\title{
Expression of CHPF modulates cell proliferation and invasion in lung cancer
}

\author{
Chengsong Cao $\mathbb{1 i}^{1,2,4 *}$, Yong Liu $\mathbb{1}^{2 *}$, Qun Wang (i) $^{2}$, Jing Zhao ${ }^{2}{ }^{2}$, Ming Shi ${ }^{3}{ }^{3}$, and \\ Junnian Zheng (ii) ${ }^{1}$ \\ ${ }^{1}$ Department of Oncology, Nanjing Medical University, Nanjing, Jiangsu, China \\ ${ }^{2}$ Department of Oncology, Xuzhou Center Hospital, Xuzhou, Jiangsu, China \\ ${ }^{3}$ Department of Oncology, Xuzhou Medical University, Xuzhou, Jiangsu, China \\ ${ }^{4}$ Xuzhou Institute of Medical Science, Xuzhou, Jiangsu, China
}

\begin{abstract}
Lung cancer is the most common malignancy worldwide and is characterized by rapid progression, aggressive behavior, frequent recurrence, and poor prognosis. The TCGA database indicates that chondroitin polymerizing factor (CHPF) is overexpressed in human lung cancer tissues compared with normal tissues and this overexpression corresponds to shorter overall survival in lung cancer patients. In this study, to investigate the function of CHPF in lung cancer, lentiviral vectors expressing CHPF shRNA were stably transduced into A549 and H1299 cells. Compared to shCtrl cells, CHPF knockdown cells had significantly reduced proliferation. Furthermore, the silencing of CHPF in A549 and H1299 cells resulted in apoptotic induction, which led to decreased colony formation. Wound healing and transwell invasion assays revealed that CHPF could positively regulate the migration of lung cancer cells. The tumorigenic role of $C H P F$ was also validated in nude mouse xenograft models. Affymetrix gene chip analysis indicated that CHPF regulated the proliferation and invasion of lung cancer cells through $C D H 1, R R M 2, M K I 67$, and TNFRSF10B. We thus highlight CHPF as a novel target for lung cancer treatment.
\end{abstract}

Key words: CHPF; Lung cancer; Proliferation; Invasion; TCGA; Affymetrix Gene Chip

\section{Introduction}

Lung cancer is one of the most lethal and common malignancies in humans, with a 5-year survival rate of $\leqslant 20 \%(1,2)$. This poor survival rate is related to an unclear pathogenesis and a lack of effective early diagnosis and treatment methods $(3,4)$. Knowledge of the molecular and cellular mechanisms that govern lung cancer development can improve future diagnostics and therapies (5-7). In recent years, kinase-targeted therapies and immune check-point inhibitors have shown increased efficacy in lung cancer treatment compared to standard chemotherapy $(6,8,9)$. However, some problems persist, including specificity, cell penetrability, and resistance. More specifically, characterized drug targets are required to overcome these barriers.

Chondroitin polymerizing factor (CHPF) is a 775 amino acid type II transmembrane protein and a member of the chondroitin synthase family (10-12). The CHPF gene is located on region $2 q 35-q 36$ of the human chromosome, spanning 4 exon regions. Studies have shown that $C H P F$ expression is upregulated in colorectal cancer (13), laryngeal cancer (14), and brain glioma (15) and that the overexpression of CHPF may be closely related to tumor occurrence and development. However, the mechanism of high CHPF expression in lung cancer development and progression has not been studied in detail. An in-depth knowledge of the molecular mechanism and related signaling pathways that govern CHPF activity may be of benefit in lung cancer treatment.

In this study, we demonstrated elevated expression of CHPF mRNA in lung cancer tissues and five lung cancer cell lines. The effects of CHPF on the proliferation and invasion of lung cancer cells were further assessed in vitro and in vivo. Microarray analysis of the gene expression changes in CHPF-silenced cells was performed to reveal the pathways regulated by CHPF.

\section{Material and Methods}

\section{Cell lines and cell culture}

The human lung cancer cell lines A549, 95-D, NCl$\mathrm{H} 1299, \mathrm{H} 1688$, and $\mathrm{NCl}-\mathrm{H} 460$ were purchased from the

Correspondence: Junnian Zheng: <chs20120830@163.com>

*These authors contributed equally to this study.

Received June 27, 2019 | Accepted February 21, 2020 
Type Culture Collection of the Chinese Academy of Sciences (China). Cells were cultured in $\mathrm{F}-12 \mathrm{~K}$ complete medium (ATCC) containing $10 \%$ fetal bovine serum (Invitrogen, USA) at $37^{\circ} \mathrm{C}$ with $5 \% \mathrm{CO}_{2}$.

\section{TCGA database analysis}

The RNA-sequencing dataset of the lung cancer cohort was downloaded from TCGA database (https:// tcga-data.nci.nih.gov/tcga/). For lung adenocarcinoma (LUAD), the expression data for CHPF in 57 paired (tumor and peri-tumor) samples and in normal $(n=59)$ and primary tumor tissues $(n=515)$ were collected and analyzed. Additionally, the survival of LUAD patients with low/ medium $(n=375)$ and high expression $(n=127)$ of CHPF was statistically analyzed.

\section{RNA isolation and quantitative real-time PCR (qRT-PCR)}

Total RNA was isolated from five lung cancer cell lines (A549, 95-D, NCl-H1299, H1688, and $\mathrm{NCl}-\mathrm{H} 460$ ) using TRIzol total RNA reagent (Pufei Biotech, China). Reverse transcription was conducted according to the instructions of M-MLV reverse transcriptase (Promega, USA) to obtain cDNA. The primers for CHPF were synthesized by Gene Chem Co. Ltd. (China). GAPDH was applied as a loading control. The sequences of the primers used in the study are as follows: GAPDH forward, 5'-TGACTTCAACAGCGA CACCCA-3' and reverse, 5'-CACCCTGTTGCTGTAGC CAAA-3'; CHPF forward, 5'-GGAACGCACGTACCAGGA G-3' and reverse, 5'-CGGGATGGTGCTGGAATACC-3'. The reactions were performed using SYBR premix Ex Taq II (Takara Biomedical Technology Co., Ltd., Japan). Relative CHPF expression was analyzed by normalizing to GAPDH. The comparative threshold cycle $\left(2^{-\triangle \triangle \mathrm{Ct}}\right.$ and $10000 / 2^{\triangle \mathrm{Ct}}$ ) equation was applied to calculate the relative CHPF mRNA expression.

shRNA lentiviral vector construction and transduction

To silence CHPF, cells were transduced with short hairpin (shRNA) lentivirus targeting the human CHPF gene (Gene ID: 79586) with pGCSIL-green fluorescent protein (GFP) for transduction rate evaluation. The shRNA sequence was as follows: shRNA-CHPF 5'-CTGGCCATGCTACTCTTTG-3'. Lentivirus lacking the shRNA insert was used as a control. A549 and H1299 cells were seeded into a 6-well plate at a density of $4 \times 10^{5}$ cells/well and transduced with shRNACHPF $\left(6 \times 10^{8} \mathrm{TU} / \mathrm{mL}\right)$ or shRNA-NC lentivirus $\left(8 \times 10^{8} \mathrm{TU} /\right.$ $\mathrm{mL}$ ). After $72 \mathrm{~h}$ of transduction, the cells were imaged under a fluorescence microscope and further selected by puromycin. Five days post-infection, CHPF silencing was verified through qRT-PCR analysis.

\section{Western blotting}

The cells were lysed with RIPA buffer for $30 \mathrm{~min}$ at $4^{\circ} \mathrm{C}$ for protein extraction after infection with lentivirus. A BCA assay was applied to determine the protein concentrations. The same amounts of protein were separated on $12.5 \%$ SDS-PAGE gels and transferred to polyvinylidene fluoride (PVDF) membranes. The membranes were incubated with anti-CCND1 (\#2978) or anti-CDH1 (\#14472) primary antibodies (Cell Signaling Technologies (CST), USA) as well as other antibodies, including those against MKI67 (ab15580), TNFRSF10B (ab8416), FOXM1 (ab180710), RRM2 (ab172476), HIF1A (ab16066) (Abcam, UK), and GAPDH (SC-32233) (Santa Cruz Biotechnology, USA). Anti-CHPF antibody (Orb127868) was purchased from Biorbyt Ltd. (UK). The membranes were then incubated with HRP-conjugated antibodies (CST, \#7076, \#7074).

\section{MTT assays}

After infection with shCtrl or shCHPF lentivirus, $1.5 \times 10^{3}$ A549 and $\mathrm{H} 1299$ cells were seeded into 96-well plates and further cultured at $37^{\circ} \mathrm{C}$ for $1-5$ days. Cells were counted using the Cellomics ArrayScan VT1 HCS automated reader (Cellomics, Inc., USA). Cell proliferation was determined by MTT assay according to the manufacturer's protocol. Briefly, after the incubation of MTT reagent with cells for $4 \mathrm{~h}$, absorbance was read at $490 \mathrm{~nm}$ on the microplate reader.

\section{Apoptosis assays}

The cells infected with shCtrl or shCHPF lentivirus were collected and labelled with annexin V-APC according to the manufacturer's protocol (eBioscience, USA). Annexin staining was measured on a FACS Calibur II sorter, and Cell Quest Research software (BD Biosciences, USA) was used for analysis.

\section{Colony forming assays}

Soft agar assays were used to assess the regulation of colony formation by CHPF at 10 days post-infection. Colonies were fixed in 4\% PFA and Giemsa-stained (Sigma-Aldrich, USA). Colonies larger than $100 \mu \mathrm{m}$ were counted.

\section{Invasion assays}

Transwell membranes pre-coated with Matrigel (BD Biosciences) were applied to evaluate the invasion effect mediated by CHPF. A total of $8 \times 10^{4}$ cells were seeded into the insert and the lower chamber of the Transwell was filled with $500 \mu \mathrm{L}$ of F-12K (Invitrogen, USA) supplemented with $10 \%$ FBS. After incubation for $24 \mathrm{~h}$, the invading cells were stained with Giemsa after the cells in the upper chamber were removed.

\section{Cell migration assays}

A wound healing assay was used to determine the migration of the cells. A total of $5 \times 10^{4}$ cells were seeded into 96 -well plates and grown to $80-90 \%$ confluency. A scratch line was made in the cell monolayer by a pipette tip. Cells were further cultured for different periods of 
time as indicated. The migrated cells were quantified using Celigo (Nexcelom, USA), and the migration rate was calculated.

\section{Xenograft models}

The animal experiment was approved by the Ethics Committee of Nanjing Medical University (No. IACUC1808010). Four-week-old female BALB/c nude mice, which were bought from Ling Chang (China), were randomly divided into two groups ( $n=6$ per group). shRNA-CHPF or normal control (NC) lentivirus-expressing A549 cells $\left(1 \times 10^{7}\right)$ were subcutaneously implanted into the right dorsal flank. The tumor volume was measured twice weekly with calipers and calculated using the following formula: $V=3.14 / 6 \times$ length $\times$ width $^{2}$. Forty days postinoculation, the tumors were excised and weighed.

\section{Microarray and data analysis}

Total RNA was extracted and purified with clean-up kits (Pufei, China). The Affymetrix Gene Chip Human Transcriptome Array 2.0 (Affymetrix Inc., USA) was used for microarray hybridization, and the chip was scanned. The signals were converted into digital information and analyzed by SAM software (Affymetrix Inc., USA). The Empirical Bayes procedure and Benjamini-Hochberg method were used to calculate and correct the P-value. Genes with a corrected $\mathrm{P}$-value less than 0.05 and an absolute value of fold-change greater than or equal to 2 were considered to be significantly differentially expressed. GO enrichment analysis of A549 cells was performed to explore the biological function of CHPF using the R software. Ingenuity Pathway Analysis (Ingenuity Systems, Inc., USA, www.ingenuity.com) was used to explore protein networks.

\section{Statistical analysis}

Data are reported as means $\pm S D$ of three replicates. Statistical analysis was carried out using the SPSS 20.0 statistical software package (IBM, USA) and Student's $t$-test. Statistical significance was accepted at $\mathrm{P}$-value $<0.05$.

\section{Results}

\section{CHPF was overexpressed in lung cancer tissues and cells}

Through the analysis of the datasets from TCGA, it was found that, compared with human normal tissues, lung cancer tissues had significant overexpression of CHPF mRNA (Figure 1A and B, Supplementary Table S1). Elevated CHPF expression corresponded to shorter overall survival in lung cancer patients (Figure 1C). CHPF mRNA levels were then assessed in lung cancer cell lines including A549, 95-D, NCl-H1299, H1688, and $\mathrm{NCl}-\mathrm{H} 460$ by qRT-PCR. The results confirmed that CHPF mRNA was expressed at high levels in all five cell lines (Figure 1D).
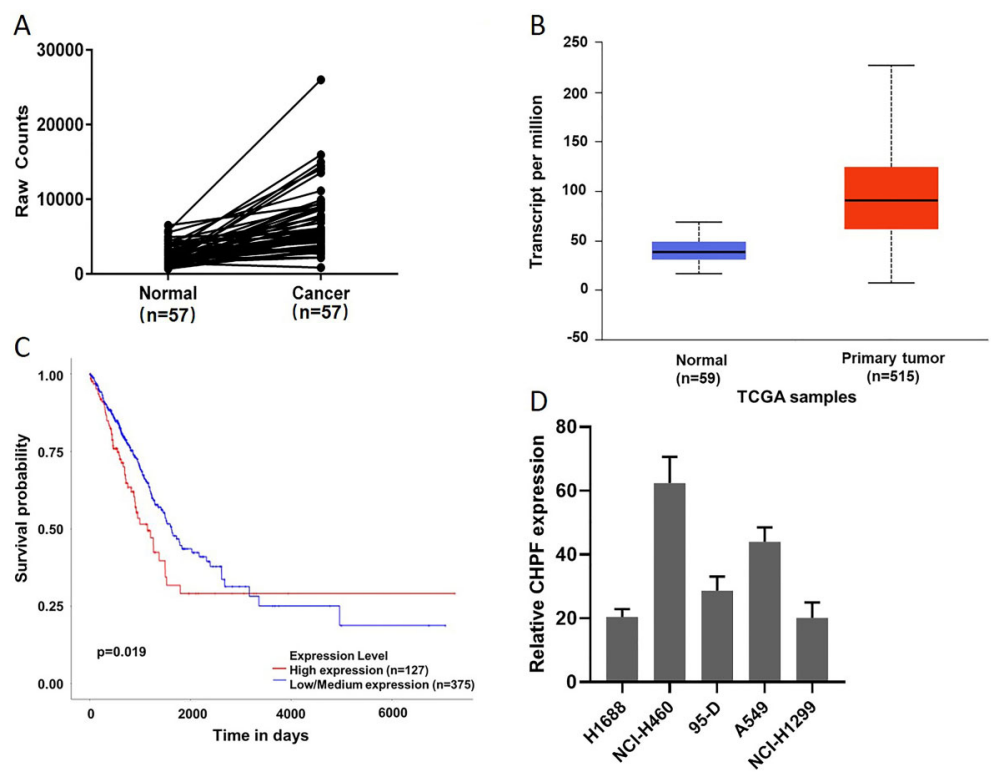

Figure 1. Chondroitin polymerizing factor $(C H P F)$ expression in lung cancer patients and lung cancer cell lines. A, CHPF mRNA expression in the lung cancer and normal tissues of samples. The solid line that shows the trend in gene expression. B, CHPF expression in human lung cancer compared to normal tissue in the TCGA database. C, Elevated CHPF expression corresponded to shorter overall survival of lung adenocarcinoma (LUAD) patients from the TCGA database. D, CHPF mRNA expression of five lung cancer cell lines was detected by qRT- PCR and calculated by the $10000 / 2^{\triangle C t}$ equation. Data are reported as median and interquartile range (B) and means $\pm S D(D)$. 
CHPF silencing inhibited lung cancer cell proliferation More than $80 \%$ of the $\mathrm{A} 549$ and $\mathrm{H} 1299$ cells that were infected with shCtrl or shCHPF lentivirus at $6 \times 10^{8} \mathrm{TU} / \mathrm{mL}$ exhibited green fluorescence, indicating successful lentiviral infection (Figure $2 A$ and $B$ ). Lentivirus-mediated RNA interference of CHPF expression was confirmed by qRT$\mathrm{PCR}$ (Figure $2 \mathrm{C}$ and $\mathrm{D}$ ) and western blotting (Figure $2 \mathrm{E}$ and $\mathrm{F}$ ). To explore the impact of CHPF on cell proliferation, A549 cells expressing shCHPF or shCtrl lentivirus were seeded into 96-well plates and Cellomics was used to analyze cell proliferation (Figure $3 \mathrm{~A}$ ). Two days post-seeding, the proliferation rates of shCHPF-infected cells were significantly lower than those of shCtrl cells in a timedependent manner (Figure 3B). Thus, CHPF silencing significantly inhibited A549 cell growth. The suppression effect of CHPF silencing on cell growth in A549 and H1299 cells was further determined by MTT assay in a five-day culture. As shown in Figure 3C, cell survival of both A549 and H1299 cells was significantly decreased, starting on the second day in the CHPF-silenced groups $(P<0.01)$. The ability to form colonies is a characteristic of malignant tumor cells. Giemsa staining was performed to explore the effect of CHPF on the colony forming ability of lung cancer cells after 15 days of culture. The number of colonies formed by A549 cells infected with shCHPF was significantly lower than the number formed by the shCtrl cells (41 \pm 3 vs $166 \pm 9$ ). Colony forming ability was also significantly lower in $\mathrm{H} 1299$ cells in the shCHPF group compared to the shCtrl control group $(22 \pm 5$ vs $108 \pm 6$, $\mathrm{P}<0.01$; Figure 3D).

\section{CHPF silencing induced cell apoptosis}

We further examined the effects of CHPF silencing on A549 and H1299 cell apoptosis by flow cytometry. The rate of apoptosis in the shCHPF A549 cells (11.88 \pm $0.50 \%$ ) was significantly higher than that in the shCtrl cells $(2.91 \pm 0.14 \%),(P<0.01$; Figure $4 A)$. Similarly, the percentage of apoptotic $\mathrm{H} 1299$ cells in the shCHPF group $(8.99 \pm 0.21 \%)$ was higher than that in the shCtrl group $(2.98 \pm 0.10 \%)(P<0.01$; Figure $4 B)$.

\section{Inhibiting CHPF repressed the invasion and migration abilities of lung cancer cells}

To evaluate the role of CHPF in lung cancer metastasis, we assessed the effects of CHPF silencing in A549 and $\mathrm{H} 1299$ cells. Wound healing assays showed that the
A

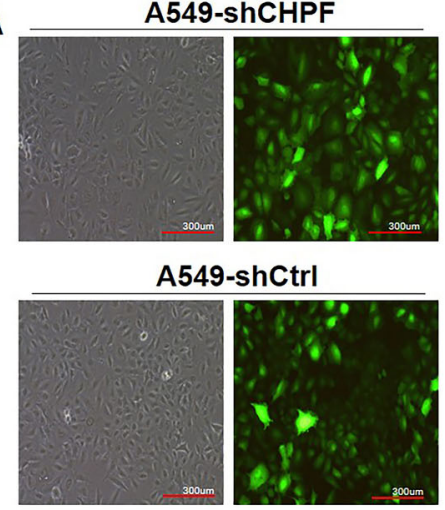

B

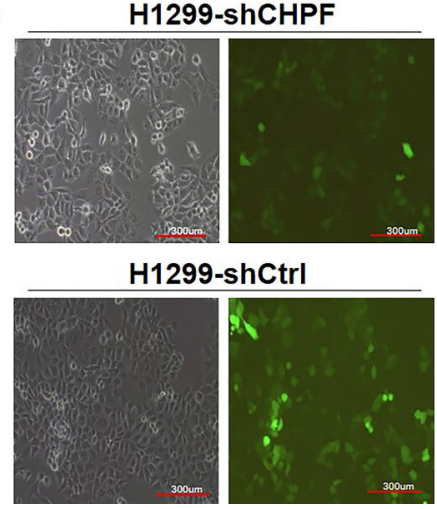

C
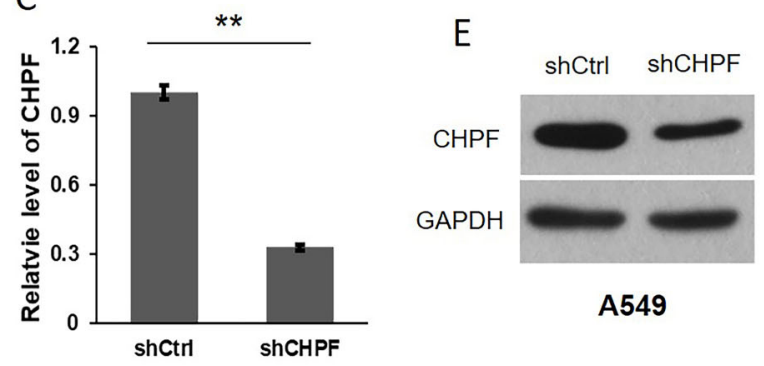

A549
D

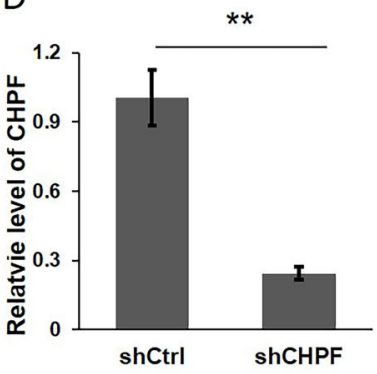

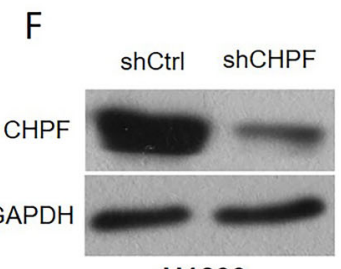

H1299

Figure 2. shRNA lentivirus transduction and confirmation. $\mathbf{A}$ and $\mathbf{B}$, Lentiviral vector expressing shCHPF or shCtrl was transduced into A549 and H1299 cells. Infection efficiency was confirmed by fluorescence microscopy at $72 \mathrm{~h}$. C to F, qRT-PCR and western blotting were used to confirm the knockdown efficiency of CHPF. ${ }^{* *} \mathrm{P}<0.01$ (Student's $t$-test). CHPF: chondroitin polymerizing factor. 
A
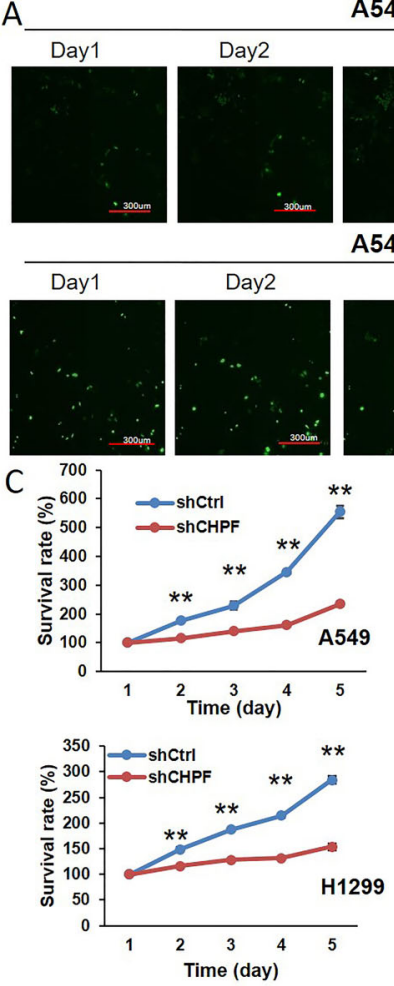

A549-shCtrl

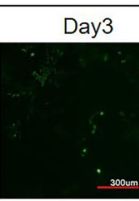

A549-shCHPF
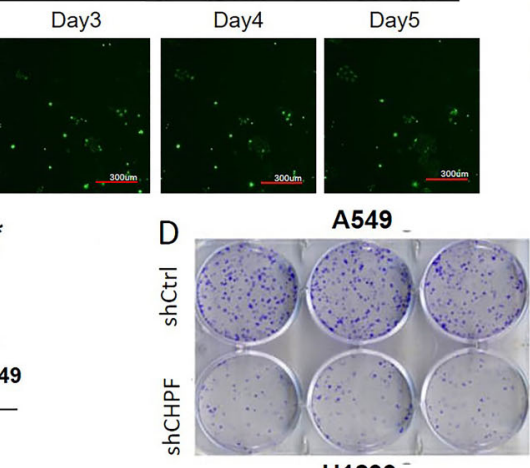

H1299

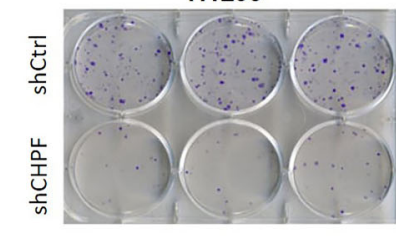

B
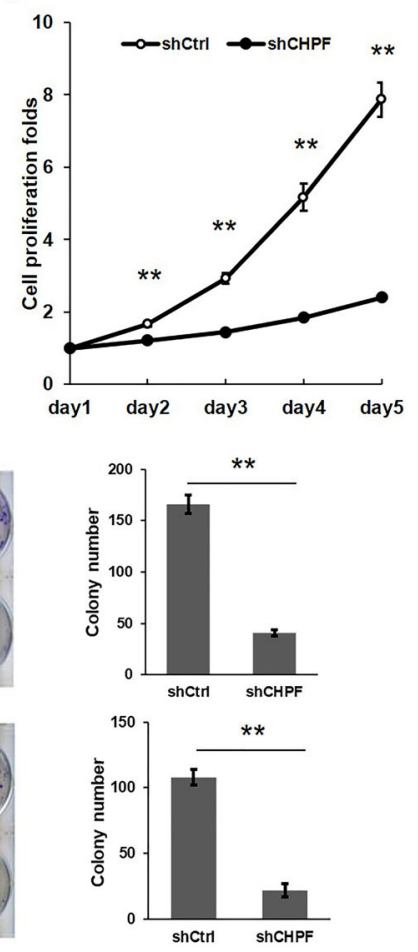

Figure 3. Effect of chondroitin polymerizing factor (CHPF) on the proliferation and colony formation of lung cancer cells. A and $\mathbf{B}$, Highcontent cell imaging was used daily to assess cell growth in A549 cells following lentivirus infection (scale bar: $300 \mu \mathrm{m}$ ). C, MTT assays displaying the survival of A549 and H1299 cells after infection with shCHPF or shCtrl for 5 days. D, Impact of CHPF silencing on the colony formation of A549 and H1299 lung cancer cells. Data are reported as means \pm SD. ${ }^{\star \star} \mathrm{P}<0.01$ (Student's $t$-test).

motility of A549 cells in the shCHPF group was significantly lower than that in the shCtrl control $(12.03 \pm 0.32 \%$ vs $37.86 \pm 4.86 \%)(P<0.01$; Figure $5 \mathrm{~A})$. The motility of H1299 cells in the shCHPF group was significantly lower than that in the shCtrl group $(32.84 \pm 5.07 \%$ vs $86.54 \pm$ $6.26 \%)(P<0.01$; Figure $5 B)$. Moreover, the invasive ability of both $\mathrm{A} 549$ and $\mathrm{H} 1299$ cells was significantly decreased following CHPF silencing as determined by the transwell assay (shCHPF: $25 \pm 1.86$ vs shCtrl: $94 \pm 0.89$ for A549 cells, shCHPF: $168 \pm 4.14$ vs shCtrl: $503 \pm 1.98$ for $\mathrm{H} 1299$ cells, $\mathrm{P}<0.01$; Figure $5 \mathrm{C}$ and $\mathrm{D})$. These data strongly support a role for CHPF in the migration and invasion of A549 and H1299 cells.

\section{CHPF silencing decreased tumorigenicity in nude mice}

A lung cancer xenograft mouse model was developed to validate the oncogenic role of CHPF. BALB/c nude mice were selected for subcutaneous injection of A549 cells infected with either shCtrl or shCHPF lentivirus. Tumor growth was continuously followed after cell implantation. Tumors were excised and their size and weight were measured (Figure $6 \mathrm{~A}$ and $\mathrm{B}$ ). After 40 days, minimal tumor growth was observed in groups injected with CHPF- silenced cells, and tumor weights were significantly lower in the CHPF-silenced group than that in the control group (Figure $6 C$ and $D$ ). These results clearly indicated that CHPF silencing inhibited lung cancer cell tumorigenicity in vivo.

\section{CHPF silencing altered oncogene expression in lung cancer cells}

Global gene expression profiling of A549 cells infected with lentivirus expressing either shCtrl or shCHPF was examined using a microarray platform. Following normalization and bioinformatic analysis, 635 differentially expressed genes (DEGs) were identified $(P<0.05$ and absolute fold-change (FC absolute) >2), including 294 upregulated and 341 downregulated genes (Figure 7A). CHPF function and pathway enrichment analyses were performed using (Ingenuity Pathway Analysis). DEGs were significantly enriched in cancer, organismal injury, gastrointestinal disease, cell death, and other pathways (Figure 7B). The interaction network of CHPF with the identified cancer associated genes was then assessed (Figure 7C). In total, 32 genes which are related to cancer development were identified to be regulated by CHPF. Among the genes, CCND1, MKI67, HIF1A, CDH1, RRM2, 
A

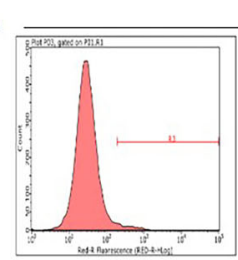

A549-shCtrl

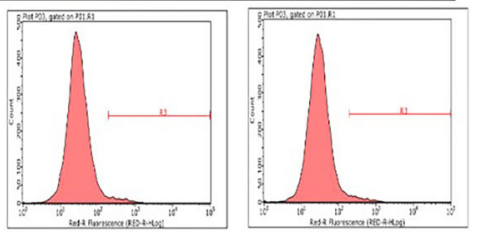

A549-shCHPF

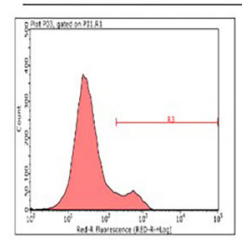

B

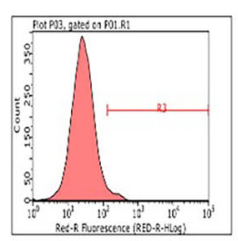

H1299-shCtrl
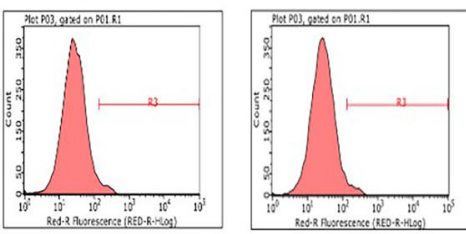

H1299-shCHPF
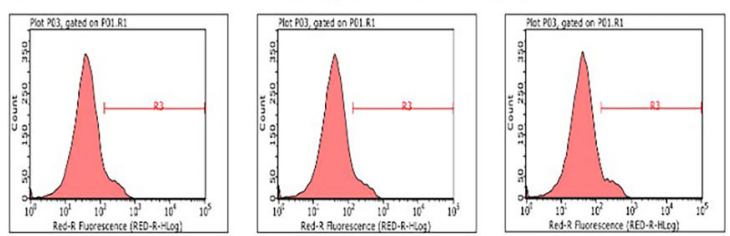
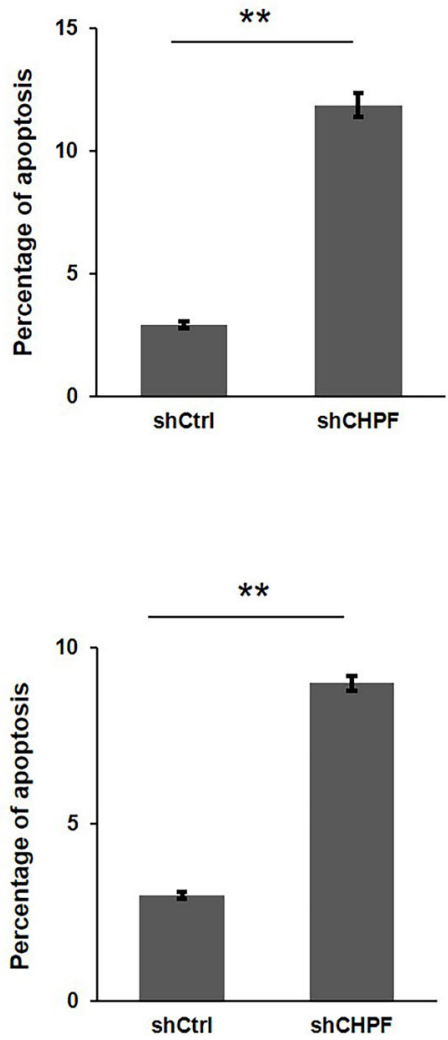

Figure 4. Effect of chondroitin polymerizing factor (CHPF) on the apoptosis of lung cancer cells. A and B, The apoptotic rates of A549 and $\mathrm{H} 1299$ cells after infection with shRNA-CHPF or shCtrl were analyzed by flow cytometry. Data are reported as means \pm SD of three independent experiments. ${ }^{* *} \mathrm{P}<0.01$ (Student's $t$-test).

and FOXM1 were significantly downregulated following CHPF silencing (Supplementary Table S2). Western blot analysis confirmed decreased $C D H 1, R R M 2$, and MKI67 expression in CHPF silenced cells. TNFRSF10B expression was significantly increased following CHPF silencing (Figure 7D).

\section{Discussion}

CHPF is a member of the chondroitin synthase family and participates in the extension of the chondroitin sulfate (CS) backbone (16). The expression and activity of CHPF is indispensable for the initiation of CS biosynthesis and the production of proteoglycan. In recent years, CHPF has been implicated in colorectal cancer, laryngeal cancer, and glioma development (13-15). However, the function of $C H P F$ in human lung cancer has remained undefined.

In this study in vitro, we found that CHPF silencing inhibited proliferation and colony formation and promoted cellular apoptosis in lung cancer cells. Moreover, CHPF silencing was also demonstrated to reduce lung cancer cell migration and invasion. The in vivo study revealed that CHPF contributed to the tumorigenicity of lung cancer cells in xenograft mouse models.

To investigate the molecular mechanism governing the ability of CHPF to promote tumorigenesis, Affymetrix gene chip analysis was used to explore changes in the expression of cancer-related genes in shCHPF and shCtrl cells. In total, 32 cancer related genes including CCND1, MKI67, HIF1A, CDH1, RRM2, and FOXM1 were subsequently identified through Ingenuity Pathway Analysis. CHPF silencing markedly downregulated the mRNA expressions of six cancer genes at the transcriptional level. The loss of expression of these genes was validated at the protein level by western blotting. RRM2 is known to be associated with tumor invasion and with the establishment of a metastatic phenotype $(17,18)$, which promotes poor overall 
A

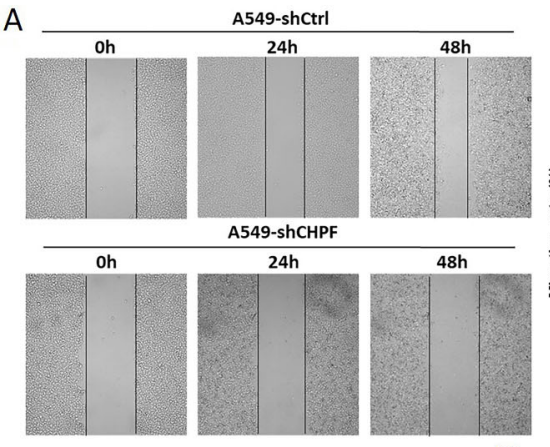

C A549-shCtrl A549-shCHPF
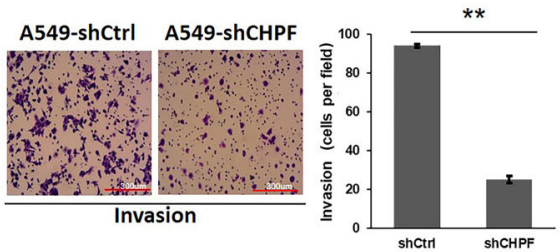

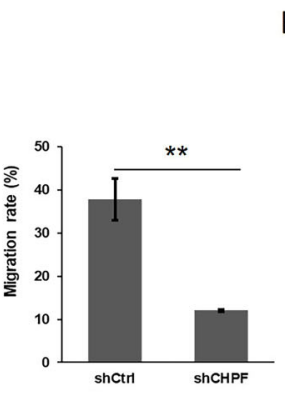

B
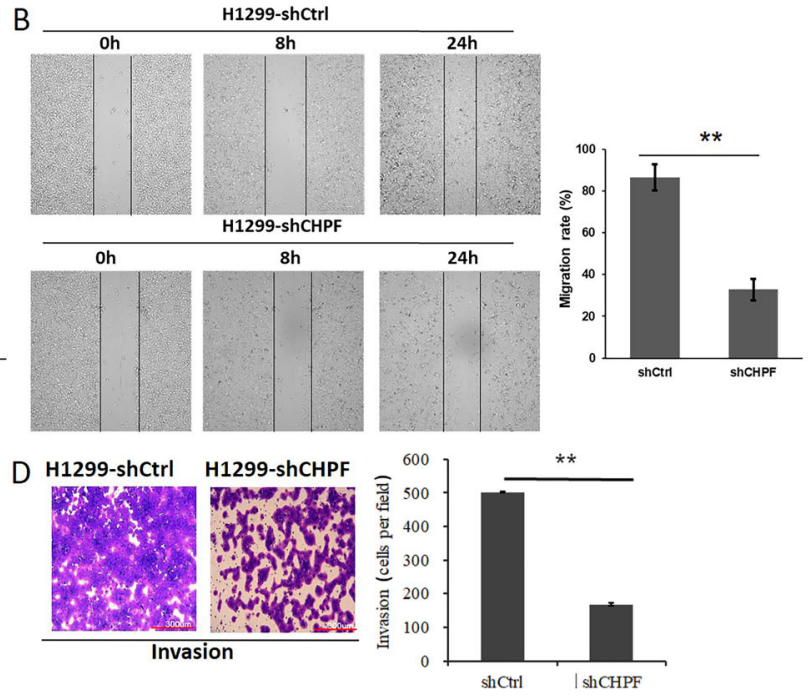

Figure 5. Effect of chondroitin polymerizing factor (CHPF) on the migration and invasion of A549 and H1299 cells. A, and B, A wound healing assay was applied to measure the number of migratory A549 and $\mathrm{H} 1299$ cells at the indicated time points. The cell invasive ability of A549 (C) and H1299 cells (D) was determined by the transwell assay after infection (scale bar: $300 \mu \mathrm{m}$ ). Data are reported as means $\pm S D$ of three independent experiments. ${ }^{\star \star} \mathrm{P}<0.01$ (Student's $t$-test).
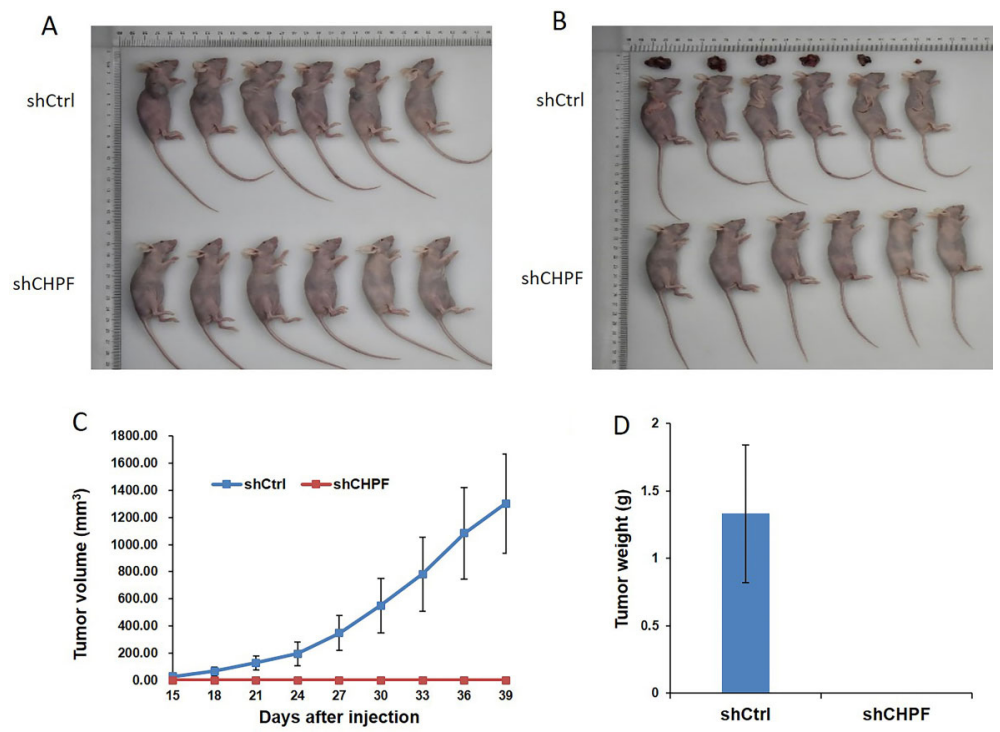

Figure 6. Effect of chondroitin polymerizing factor (CHPF) on tumorigenicity in nude mice. A, Equal numbers of CHPF silenced (shCHPF) or control A549 cells were subcutaneously administered to nude mice. B, Excised tumors were recorded. The volume (C) and weight $(D)$ of tumors were measured $(n=6)$. Data are reported as means $\pm S D$.

and progression-free survival $(19,20)$. In our study, it was validated that $C H P F$ downregulation was associated with decreased RRM2 levels.

The MKI67 labeling index also correlates with tumor growth rates (21), histologic stage (22), and tumor recurrence (23). Elevated MKI67 expression indicates rapid cancer progression and poor prognosis. According to our investigation, CHPF silencing decreased MKI67 expression, which may contribute to the effects of CHPF depletion on malignancy. Death receptor (DR) signaling forms one arm of the extrinsic apoptotic processes (24). TNFrelated apoptosis-inducing ligand (TRAIL) and its receptor DR5 (TNFRSF10B) promote tissue damage in somatic cells (25). It was suggested that CHPF silencing in A549 


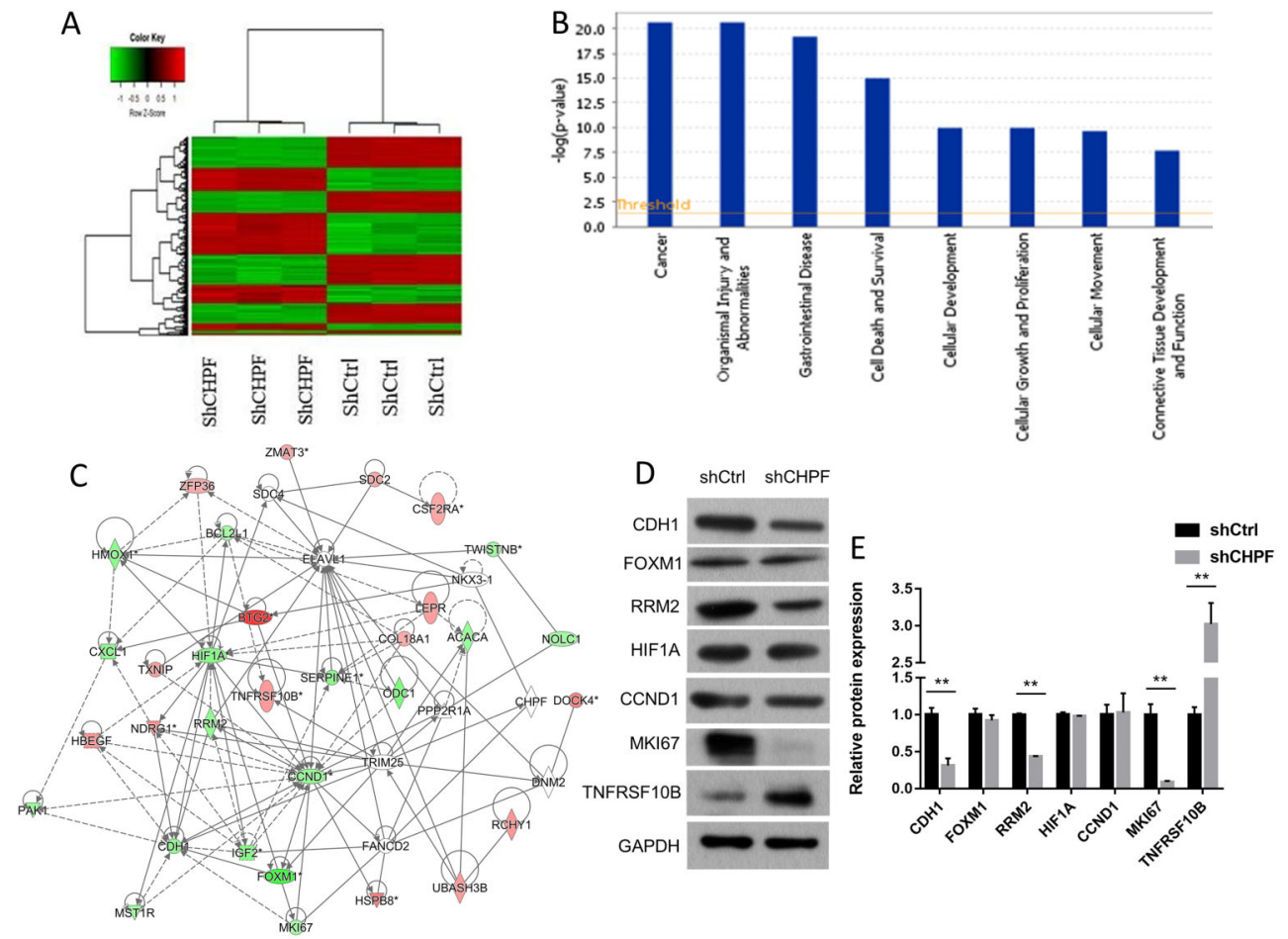

Figure 7. Effect of chondroitin polymerizing factor (CHPF) on global gene expression. A, Heatmap representation of 635 differentially expressed genes (DEGs) in A549 cells infected with shCHPF or shCtrl. $\mathrm{P}<0.05$ and fold-change $>2$ (red: upregulated genes; green: downregulated genes). B, Functional pathway enrichment of the DEGs was analyzed via Ingenuity Pathway Analysis. The $\mathrm{X}$-axis represents the pathway. Statistical significance is shown on the $y$-axis and represents the inverse log of the P-value. C, The network was built on the basis of microarray data of lung cancer cells. Red represents upregulation and green represents downregulation. $\mathbf{D}$, Western blotting analysis was applied to evaluate the expression of CDH1, FOXM1, RRM2, HIF1A, CCND1, MKI67, and TNFRSF10B in shCHPF and shCtrl lung cancer cells. Data are reported as means \pm SD. ${ }^{*} \mathrm{P}<0.01$ (Student's $t$-test).

cells may enhance apoptosis through the upregulation of TNFRSF1OB. The CDH1 gene is believed to be related to tumor proliferation (26). The above data suggest that the effects of CHPF silencing on lung cancer cell proliferation may be related to the downregulation of $C D H 1$. Additionally, using Ingenuity Pathway Analysis, we identified numerous genes regulated by CHPF centralized networks that need further investigation.

In summary, we found that the expression of CHPF in lung cancer tissues was higher than that in normal lung tissues. Elevated CHPF expression corresponded to shorter overall survival. CHPF was found to promote lung cancer cell proliferation, migration, and invasion in vitro and tumorigenesis in vivo. It is speculated that CHPF mediated the above effects through the dysregulation of cancer-related genes, including CDH1, RRM2, MKI67,

\section{References}

1. Siegel RL, Miller KD, Jemal A. Cancer statistics, 2018. CA Cancer J Clin 2018; 68: 7-30, doi: 10.3322/caac.21442. and TNFRSF10B. These findings provide a proof-ofconcept that revealed the cellular roles of CHPF and its contribution to lung cancer malignancy. This highlighted CHPF as a potential therapeutic target for the clinical treatment of lung cancer.

\section{Supplementary Material}

Click here to view [pdf].

\section{Acknowledgments}

This work was supported by Jiangsu Young Medical Talent Fund (QNRC 2016379) and Xuzhou Science and Technology Bureau Applied Basic Research Program (KC18035).

2. Wong MCS, Lao XQ, Ho KF, Goggins WB, Tse SLA. Incidence and mortality of lung cancer: global trends and 
association with socioeconomic status. Sci Rep 2017; 7: 14300-14308, doi: 10.1038/s41598-017-14513-7.

3. Testa U, Castelli G, Pelosi E. Lung Cancers: molecular characterization, clonal heterogeneity and evolution, and cancer stem cells. Cancers (Basel) 2018; 10. pii: E248, doi: 10.3390/cancers 10080248 .

4. Tsvetkova E, Goss GD. Drug resistance and its significance for treatment decisions in non-small-cell lung cancer. Curr Oncol 2012; 19: S45-S51, doi: 10.3747/co.19.1113.

5. Hubers AJ, Prinsen CF, Sozzi G, Witte BI, Thunnissen E. Molecular sputum analysis for the diagnosis of lung cancer. Br J Cancer 2013; 109: 530-537, doi: 10.1038/bjc.2013.393.

6. Politi K, Zakowski MF, Fan PD, Schonfeld EA, Pao W, Varmus HE. Lung adenocarcinomas induced in mice by mutant EGF receptors found in human lung cancers respond to a tyrosine kinase inhibitor or to down-regulation of the receptors. Genes Dev 2006; 20: 1496-1510, doi: 10.1101/gad.1417406.

7. Chen Z, Fillmore CM, Hammerman PS, Kim CF, Wong KK. Non-small-cell lung cancers: a heterogeneous set of diseases. Nat Rev Cancer 2014; 14: 535-546, doi: 10.1038/nrc3775.

8. Roskoski R Jr. Anaplastic lymphoma kinase (ALK) inhibitors in the treatment of ALK-driven lung cancers. Pharmacol Res 2017; 117: 343-356, doi: 10.1016/j.phrs.2017.01.007.

9. Tanvetyanon T, Gray JE, Antonia SJ. PD-1 checkpoint blockade alone or combined PD-1 and CTLA-4 blockade as immunotherapy for lung cancer? Expert Opin Biol Ther 2017; 17: 305-312, doi: 10.1080/14712598.2017.1280454.

10. Siebert JR, Conta Steencken A, Osterhout DJ. Chondroitin sulfate proteoglycans in the nervous system: inhibitors to repair. Biomed Res Int 2014; 2014: 845323, doi: 10.1155/ 2014/845323.

11. Kitagawa H, Uyama T, Sugahara K. Molecular cloning and expression of a human chondroitin synthase. $J$ Biol Chem 2001; 276: 38721-38726, doi: 10.1074/jbc.M106871200.

12. Kitagawa H, Izumikawa T, Uyama T, Sugahara K. Molecular cloning of a chondroitin polymerizing factor that cooperates with chondroitin synthase for chondroitin polymerization. J Biol Chem 2003; 278: 23666-23671, doi: 10.1074/jbc.M3 02493200.

13. Kalathas D, Theocharis DA, Bounias D, Kyriakopoulou D, Papageorgakopoulou N, Stavropoulos MS, et al. Chondroitin synthases I, II, III and chondroitin sulfate glucuronyltransferase expression in colorectal cancer. Mol Med Rep 2011; 4: 363-368, doi: 10.3892/mmr.2011.431.

14. Kalathas D, Triantaphyllidou IE, Mastronikolis NS, Goumas PD, Papadas TA, Tsiropoulos G, et al. The chondroitin/ dermatan sulfate synthesizing and modifying enzymes in laryngeal cancer: expressional and epigenetic studies. Head Neck Oncol 2010; 2: 27-34, doi: 10.1186/1758-3284-2-27.
15. Fan YH, Xiao B, Lv SG, Ye MH, Zhu XG, Wu MJ. Lentivirusmediated knockdown of chondroitin polymerizing factor inhibits glioma cell growth in vitro. Oncol Rep 2017; 38: 1149-1155, doi: 10.3892/or.2017.5731.

16. Mizumoto S, Yamada S, Sugahara K. Human genetic disorders and knockout mice deficient in glycosaminoglycan. Biomed Res Int 2014; 2014: 495764, doi: 10.1155/ 2014/495764.

17. Reichard P. Ribonucleotide reductases: the evolution of allosteric regulation. Arch Biochem Biophys 2002; 397: 149155, doi: 10.1006/abbi.2001.2637.

18. Tanaka H, Arakawa H, Yamaguchi T, Shiraishi K, Fukuda S, Matsui $\mathrm{K}$, et al. A ribonucleotide reductase gene involved in a p53-dependent cell-cycle checkpoint for DNA damage. Nature 2000; 404: 42-49, doi: 10.1038/35003506.

19. Liu X, Zhang H, Lai L, Wang X, Loera S, Xue L, et al. Ribonucleotide reductase small subunit M2 serves as a prognostic biomarker and predicts poor survival of colorectal cancers. Clin Sci (Lond) 2013; 124: 567-578, doi: 10.1042/ CS20120240.

20. Farrell JJ, Moughan J, Wong JL, Regine WF, Schaefer P, Benson $A B$ 3rd, et al. Precision medicine and pancreatic cancer: a gemcitabine pathway approach. Pancreas 2016; 45: 1485-1493, doi: 10.1097/MPA.0000000000000710.

21. Maeda T, Takenaka K, Adachi E, Matsumata T, Shirabe K, Honda $\mathrm{H}$, et al. Small hepatocellular carcinoma of single nodular type: a specific reference to its surrounding cancerous area undetected radiologically and macroscopically. J Surg Oncol 1995; 60: 75-79, doi: 10.1002/jso.2930 600202.

22. $\mathrm{Ng} \mathrm{IO}, \mathrm{Na} \mathrm{J}$, Lai EC, Fan ST, Ng M. Ki-67 antigen expression in hepatocellular carcinoma using monoclonal antibody MIB1. A comparison with proliferating cell nuclear antigen. Am J Clin Pathol 1995; 104: 313-318, doi: 10.1093/ ajcp/104.3.313.

23. Luo $\mathrm{Y}$, Ren F, Liu $\mathrm{Y}$, Shi $\mathrm{Z}$, Tan $\mathrm{Z}$, Xiong $\mathrm{H}$, et al. Clinicopathological and prognostic significance of high Ki-67 labeling index in hepatocellular carcinoma patients: a meta-analysis. Int J Clin Exp Med 2015; 8: 10235-10247.

24. Orzalli MH, Kagan JC. Apoptosis and necroptosis as host defense strategies to prevent viral infection. Trends Cell Biol 2017; 27: 800-809, doi: 10.1016/j.tcb.2017.05.007.

25. Davidson S, Crotta S, McCabe TM, Wack A. Pathogenic potential of interferon alphabeta in acute influenza infection. Nat Commun 2014; 5: 3864, doi: 10.1038/ncom $\mathrm{ms} 4864$.

26. Dong LL, Liu L, Ma CH, Li JS, Du C, Xu S, et al. E-cadherin promotes proliferation of human ovarian cancer cells in vitro via activating MEK/ERK pathway. Acta Pharmacol Sin 2012; 33: 817-822, doi: 10.1038/aps.2012.30. 